\section{Aetiology and pathogenesis of cranial cruciate ligament rupture in cats by histological examination}

\author{
Marlis Wessely ${ }^{1}$, Sven Reese $^{2}$ and Eva Schnabl-Feichter ${ }^{1}$
}

Journal of Feline Medicine and Surgery 2017, Vol. 19(6) 631-637

(c) The Author(s) 2016

Reprints and permissions: sagepub.co.uk/journalsPermissions.nav DOI: $10.1177 / 1098612 \times 16645142$ journals.sagepub.com/home/jfms

This paper was handled and processed by the European Office (ISFM) for publication in JFMS

\title{
(S)AGE
}

\begin{abstract}
Objectives The aim of this study was to examine histologically intact and ruptured cranial cruciate ligaments in cats, in order to evaluate whether degeneration is a prerequisite for rupture.

Methods We performed a histological examination of 50 intact and 19 ruptured cranial cruciate ligaments in cadaver or client-owned cats, respectively, using light microscopy. Cats with stifle pathology were further divided into five age groups in order to investigate the relationship of changes in the ligament with lifespan. Cats with ruptured cranial cruciate ligaments were divided into two groups according to medical history (with presumed history of trauma or without any known history of trauma) in order to investigate the relationship of ligament rupture with a traumatic event. Data from 200 healthy cats were selected randomly and reviewed to make a statistical comparison of cats with and without cranial cruciate ligament rupture (reference group).

Results On histological examination, the intact cranial cruciate ligaments showed basic parallel arrangement of the collagen fibres, with no relation to age. While cats of a more advanced age showed fibrocartilage in the middle of the cranial cruciate ligament - a likely physiological reaction to compression forces over the lifespan - degenerative changes within the fibrocartilage were absent in all cases, regardless of age or rupture status. Cats suffering from cranial cruciate ligament rupture without history of trauma were significantly older than cats in the reference group. Conclusions and relevance This study showed that differentiation of fibrocartilage in the middle of the cranial cruciate ligament is likely a physiological reaction to compressive forces and not a degenerative change associated with greater risk of rupture in advanced age. This finding in cats is distinct from the known decrease in differentiation of fibrocartilage in dogs with cranial cruciate ligament rupture. Furthermore, the histological examination of cats revealed no other signs of degeneration in the cranial cruciate ligaments. Thus, degeneration is likely not an aetiological factor for cranial cruciate ligament rupture in cats.
\end{abstract}

Accepted: 25 March 2016

\section{Introduction}

Reports of cranial cruciate ligament (CCL) rupture (CCLR) in cats are remarkably less frequent than for dogs. ${ }^{1}$ While the exact aetiology of canine CCL disease (CCLD) remains undefined and controversial, 2,3 data from the collective studies have suggested that various factors can promote degeneration of the CCL and increase the risk of developing CCLD. ${ }^{4,5}$

At the microscopic level, ligaments consist of cells that are generally arranged in longitudinal rows that are separated by collagen fibres, proteoglycan threads and granules. The collagen fibres are made up of individual collagen fibril units, and the larger surface area of the packed units is amenable to ultrastructure analysis by light microscopy. ${ }^{6}$ The collagen fibres themselves further pack together to form fibre bundles, and those bundles pack together to form fascicles. Collections of fascicles form the ligaments, which are themselves wrapped up in a surface connective tissue layer known as the epitenon or epiligament. ${ }^{7}$

${ }^{1}$ Department for Companion Animals and Horses, University Clinic for Small Animals, Small Animal Surgery, University of Veterinary Medicine, Vienna, Austria

2Department of Anatomy, Ludwig-Maximilians-University, Munich, Germany

\section{Corresponding author:}

Marlis Wessely DVM, Department for Companion Animals and Horses, University Clinic for Small Animals, Small Animal Surgery, University of Veterinary Medicine, Veterinärplatz 1, 1210 Vienna, Austria

Email: marlis.wessely@vetmeduni.ac.at 
The histological features of the CCL in dogs are extensively reported in the literature, ${ }^{8-12}$ and the data have helped advance studies of the underlying pathogenic mechanisms of CCLD. The histological changes that have been thus far implicated in CCLD are degeneration and chondroid metaplasia of the extracellular matrix, decreased ligamentocyte density, disorganisation of collagen fibres and phenotypic changes in ligamentocytes. ${ }^{11}$ However, normally, dogs begin to show signs of loss of the typical ligament structure at an early age ( $2-5$ years old), and the presence of shattered or ruptured collagen fibres is common. ${ }^{12}$ Specific signs of degeneration within the fibrocartilage cells (eg, lipid droplets and calcification), marked cartilage substance and cartilage cells with well-demarcated capsules are also observed regularly. ${ }^{12}$

To our knowledge, there are no such related data, from large groups of cats, with regard to histological examination of the cruciate ligaments. A report by Harasen, ${ }^{13}$ which provides data from histopathological examination of one feline CCL with a non-traumatic rupture, represents the single publication providing insight into CCLR in cats and indicated that degeneration might be part of the aetiopathogenesis.

It is unclear whether the pathogenesis of CCLR in felines is similar to that in canines, or if feline patients are unique in that trauma plays a much greater role in the disease process. ${ }^{14}$ In the literature on feline patients, reports of polytraumatic stifle injuries are more common then those of isolated ruptures of the CCL.13,15,16 Recently, however, an increasing number of reports have been published describing CCLR in indoor cats (ie, cats with less likelihood of a traumatic event); ${ }^{13,15}$ overview of these cases reveals some parallels with the reports of degenerative CCLR in canines, namely the features of older age, overweight, small breed and a lack of traumatic history. ${ }^{13}$ Thus, a proportion of cats may be affected by degeneration of the ligament, which could precede rupture.

The aims of this study were therefore: (1) to evaluate changes in the feline CCL with respect to lifespan; (2) to determine the differential features of intact and ruptured CCL as determined by light microscopy-based histological examination, with specific focus on whether degeneration is an aetiological or contributing factor; and (3) to investigate whether trauma may contribute to risk of CCLR. Our hypothesis was that, similar to the dog, degeneration of the CCL would predispose the cat to a rupture during a minimal traumatic event.

\section{Materials and methods}

\section{Animals}

Cats with CCLR Nineteen cats (19 ligaments) with CCLR that were treated surgically between September 2010 and May 2012 were included in the study and grouped according to duration of lameness. Cats with lameness for $<2$ weeks and a presumed history of trauma (including outdoor cats) were assigned to group A ( $\mathrm{n}=$ 15). The features of group $A$ were mean age at diagnosis of $7.7 \pm 2.9$ years, neutered male:spayed female ratio of $7: 15$, mean weight of $4.9 \pm 1.3 \mathrm{~kg}$ and included domestic shorthairs $(n=12)$, Maine Coons $(n=2)$ and British Shorthairs $(n=1)$. Cats with lameness for $>3$ weeks and a history of no trauma (including indoor cats) were assigned to group $B(n=4)$. The features of group $B$ were mean age of $11.7 \pm 1.5$ years, neutered male:spayed female ratio of $1: 3$, mean bodyweight of $4.4 \pm 0.25 \mathrm{~kg}$; all cats in this group were domestic shorthairs $(n=4)$.

Among the 19 cats in groups A and B, surgical treatment provided stabilisation of the stifle joints by lateral fabellotibial suture $(n=17)$ or by tibial plateau levelling osteotomy $(\mathrm{n}=2)$.

Cats without CCLR Fifty ligaments without CCLR were obtained from 26 cats that had either died or were euthanased for causes unrelated to the study topic (immediate post-mortem storage at $-20^{\circ} \mathrm{C}$ ) and which had macroscopically intact cranial and caudal cruciate ligaments; no considerations were taken in account with regard to age, sex, weight or breed. During the procedure to harvest the ligaments from the stifle, two ligaments sustained damage that caused their exclusion from the analysis. The features of this non-CCLR group were mean age of $6.3 \pm 5.6$ years, neutered/male total:spayed/ female total ratio of 12/16:6/10 and mean weight of 3.5 $\pm 2.1 \mathrm{~kg}$. These cats were grouped according to age: group $1,<0.6$ years $(\mathrm{n}=6)$; group $2,0.6-3$ years $(\mathrm{n}=4)$; group 3, 3-7 years $(\mathrm{n}=6)$; group $4,7-12$ years $(\mathrm{n}=5)$; group $5,>12$ years $(n=5)$.

Control group for statistical comparison Data from 200 healthy cats were selected randomly from the general clinic database and reviewed to make a statistical comparison of age, weight and sex of the groups of cats with and without CCLR. The features of this randomly selected group were mean age of $7.6 \pm 3.7$ years, male:female ratio of 113:87 and mean weight of $4.8 \pm 1.5 \mathrm{~kg}$.

\section{Specimen preparation}

For CCLR specimens, the remnants of the damaged CCLs were removed during the surgical treatment and fixed in $4 \%$ formalin solution. For non-CCLR specimens, the cadavers were thawed to room temperature, and the ligaments were carefully harvested and immediately transferred into a $4 \%$ formalin solution. For experimental analysis, all samples were first washed in tap water for $12 \mathrm{~h}$ to remove the residual formalin, after which they were fixed by passage through an ascending alcohol series and embedded in liquid paraffin (Paraffin Richard Allan-Scientific, Company Microm). All samples were incubated overnight (heated to a temperature of $63^{\circ} \mathrm{C}$ ) and then cut with a rotary microtome (Rotary microtome 
typ HM 360; Microm International) into $5 \mu \mathrm{m}$ slices for staining with haematoxylin and eosin.

\section{Histological examination}

All histological examinations were carried out with light microscopy, using a Microscop Typ Axioscop 2plus (Zeiss) equipped with a camera (ProgRes Jenoptik) for photographic documentation. To distinguish the proteoglycans within the fibrous tissue, Astra blue staining was performed. To distinguish the glycoproteins, periodic acid-Schiff (PAS) staining was performed. Standard histological features of chondroid metaplasia, extracellular matrix alteration and mineralisation were investigated, as well as the morphology of ligamentocytes and their transformation into chondroid cells.

\section{Statistical analysis}

Data related to age, sex and weight were expressed as means \pm SD and as range. Comparison of the epidemiological data of groups A and B and of the control group for statistical comparison were performed with the Microsoft Excel effective size calculator to determine the effective size of Cohen (Cohen's d) (supplementary material) and with SPSS 20 software (IBM) to assess independent variables with the Student's $t$-test. Differences were considered statistically significant when $P<0.05$. For Cohen's d, an effect size of $<0.3$ was considered to be a small effect, 0.3 0.5 a medium effect and $>0.8$ a large effect.

\section{Results}

Cats with CCLR

Epidemiological data: group A The duration of lameness in this group was $<2$ weeks (range 1-14 days). Five pet owners were able to provide details of the cause of lameness: jumping off the scratcher $(n=2)$, chasing after a bird $(n=1)$, fighting with a dog $(n=1)$ and jumping off a cupboard $(n=1)$. All of these cats presented lameness immediately after the traumatic event, and 2/5 had an additional trauma to the medial collateral ligament and the medial meniscus. All other cats in group A $(n=10)$ suffered from an isolated rupture of the CCL. The remaining 10 pet owners stated that their cats returned home with acute lameness after being outside. All 10 cats had been diagnosed with an isolated rupture of the CCL.

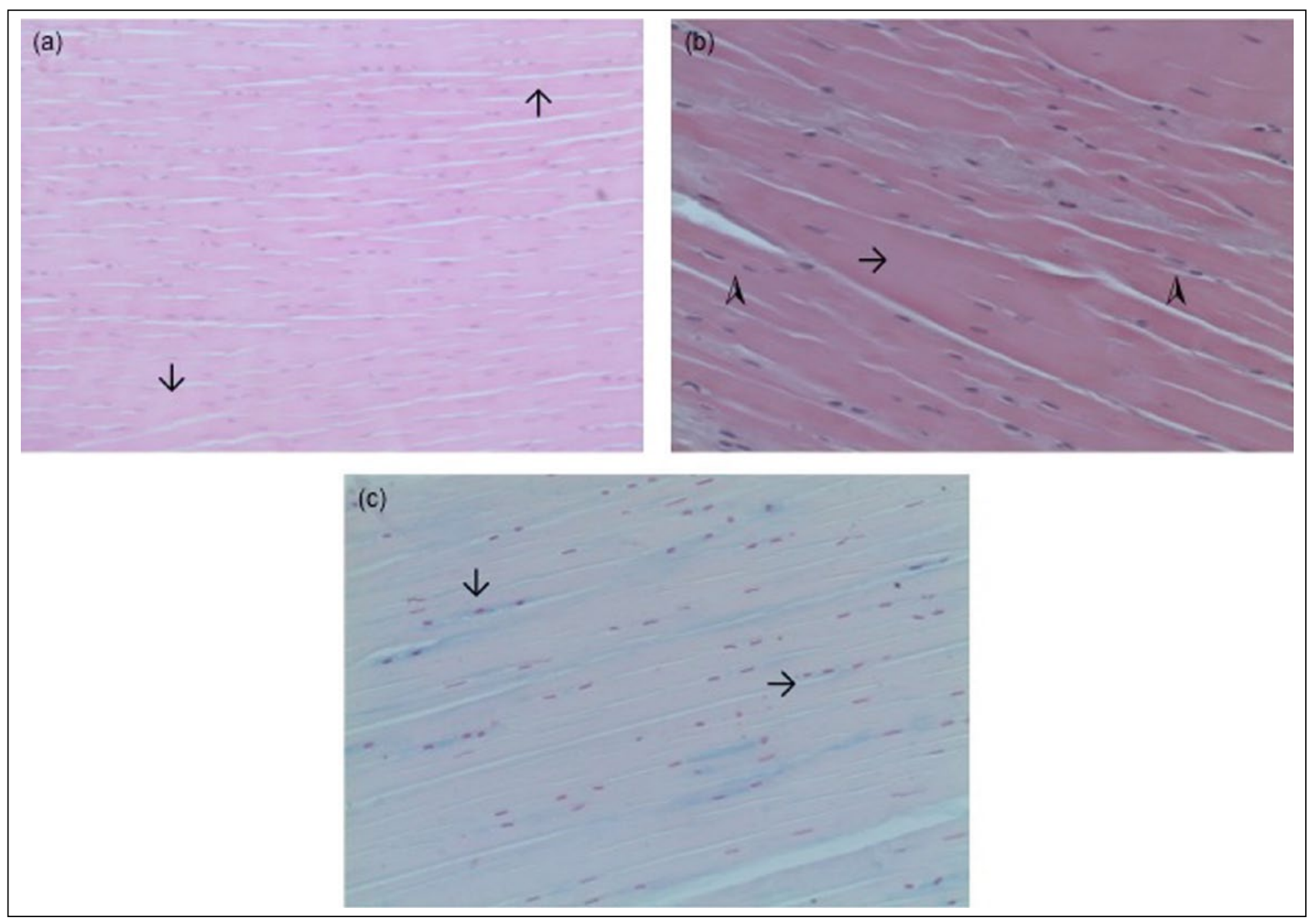

Figure 1 Microscopic anatomy of intact cranial cruciate ligaments in different age groups. (a) Four-month-old cat, weighing $1.2 \mathrm{~kg}$, haematoxylin and eosin (H\&E) staining, parallel collagen fibres (arrows); (b) 2-year-old cat, weighing $7 \mathrm{~kg}, \mathrm{HE}$ staining, increase in collagen (arrow), fibrocytes in longitudinal rows (arrowheads); (c) 5-year-old cat, weighing $2.75 \mathrm{~kg}$, Astra blue staining, rounder cells in chains (arrows) 
The cats in group A were all treated by surgery, performed at postdiagnosis day $2(n=7)$, day $7(n=5)$ or day $14(\mathrm{n}=3)$.

Epidemiological data: group $B$ The duration of lameness in this group was $>3$ weeks (range 3-9 weeks). None of these cats had a history of trauma and all resided exclusively indoors. One owner described noticing a varying degree of lameness affecting both hindlimbs over a period of $>3$ weeks. Two owners described noticing alternating signs of lameness over a period of several weeks, which varied in degree and duration. The remaining owner described noticing lameness of one hindlimb over a period lasting $>4$ weeks.

The mean body weight of the cats in group $B$ was not significantly different from that of the cats in group A; thus, cats suffering from CCLR were not heavier than healthy cats. However, the mean age of the cats in group A, which was very similar to that of the control group (Cohen's $\mathrm{d}=0.03$ ), was significantly lower than in group $B$ cats (Cohen's d $=1.48)$.

\section{Microscopic anatomy determined by histological examination \\ Cats without CCLR}

- Group 1 ( $<0.6$ years) The CCLs of cats of a younger age were dominated by parallel collagen fibres and elaborately shaped ligament cells positioned in longitudinal rows (Figure 1a). The primary fibre bundles were narrow, which precluded classification of the secondary fibre bundles. The extracellular matrix showed a negative PAS reaction primarily and no staining with Astra blue. The fibrocartilage could not be distinguished in this age group.

- Group 2 (0.6-3 years) Compared with group 1, the connective tissue was dense and the primary fibre bundles were distinctly widened, owing to the increase in collagen that normally occurs between these ages. Classification of secondary fibre bundles was now possible; most of the cells showed typical appearance of fibrocytes (elaborate shape, longitudinal rows), and only the distance between the cells appeared to have enlarged (Figure $1 b$ ). In one cat, the fibrocytes showed a slight conversion in chondroid shaped cells, with the cells attaining a more rounded shape with a centrally positioned nucleus and marked cytoplasm. Strikingly, this cat was the second heaviest $(7 \mathrm{~kg})$ in this current investigation.

- Group 3 (3-7 years) The primary fibre bundles appeared slightly narrower but remained sufficiently definable with a distinct fibre orientation. In contrast to the previous groups, the cells were rounder, still with the central positioning of the nuclear compartment but with a wider cytoplasmic compartment; the cells themselves were also laid out in longitudinal chains, which showed a marked PAS reaction and staining with Astra blue (Figure 1c).

- Group 4 (7-12 years) The primary fibre bundles were sufficiently definable and showed a distinct fibre orientation. The cell shape was slightly rounder than the other groups and somewhat flattened. Compared with group 3, the cytoplasmic compartment was wider and the longitudinal chains of cells were shorter. Some cells were only visible as shaded fragments, suggesting cell death. Similar to group 3, marked PAS reaction and increased Astra blue stain appeared around the cells (Figure 2a).

- Group 5 (>12 years) The basic parallel fibred structure of collagen was retained in the oldest cats. However, the primary and secondary fibre bundles were slightly narrower, but not to an extent that precluded their distinction. The cell shape was the most rounded and flattened of all groups thus far, with a large central nucleus and a wide cytoplasmic compartment. Longitudinal chains of cells, as well as small group formations, were visible. PAS-positive areas and further increased Astra blue staining was also present (Figure $2 b$ ).

\section{Cats with CCLR}

- Group $A$ The basic parallel fibred structure of the collagen bundles was generally retained in the intact area of the ligament. In 2/6 ligaments, however, there was a marked loss of basic parallel fibred structure. The primary fibre bundles could be readily identified. In the ruptured area of the ligament, though, the collagen fibres showed a distinct rolled-up conformation, with no clear fibre structure. The cells were of chondroid shape, with a central nucleus and wide cytoplasmic compartment; the cells were primarily arranged in small groups (2-3 cells), but no longitudinal chains or rows of cells were present.

PAS-positive areas were seen primarily around the chondroid cells, with some cells being resistant to the dye, indicating necrotic cell death. In 2/6 ligaments there was evidence of cartilage cells, lying in small groups. However, these cartilage cells showed no signs of degeneration (eg, lipid droplets and calcification) (Figure 3).

- Group B Evidence of the original ligament structure was absent. Very occasionally, fragments of collagen fibres were present. Wound healing processes and vascularisation were observed in the foreground, and the defective areas appeared to be filled up with replacement and adipose tissue (Figure 4). In some specimens, no ligament stump remained. 


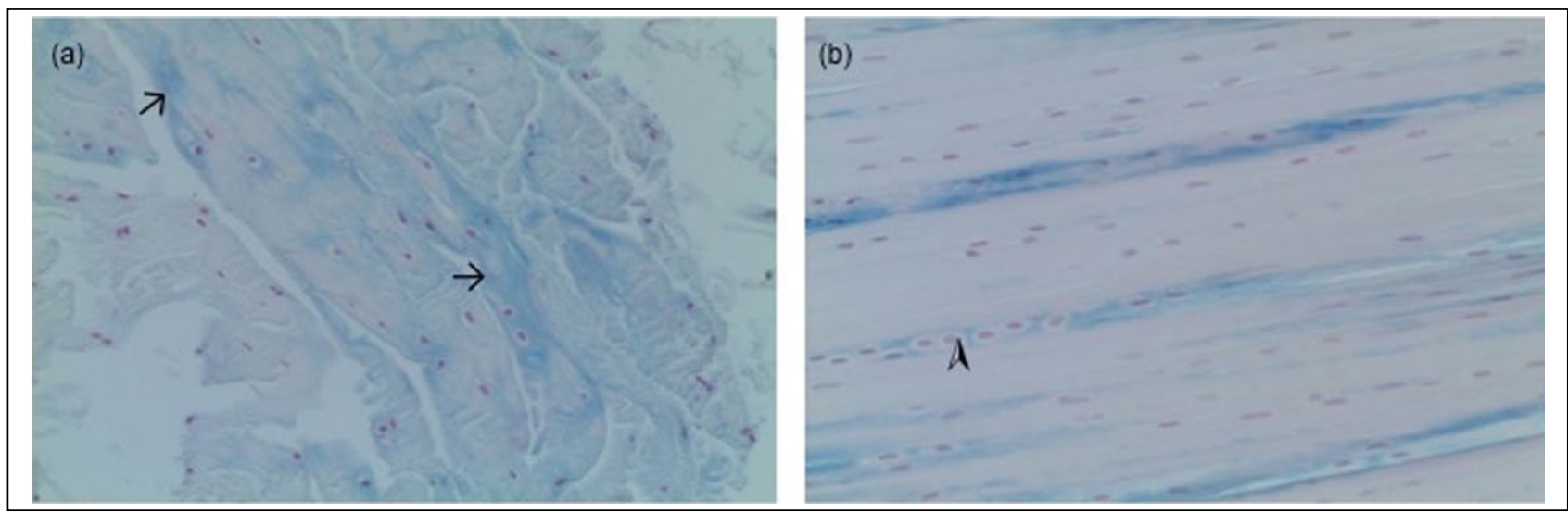

Figure 2 Microscopic anatomy of intact cranial cruciate ligaments in different age groups. (a) Ten-year-old cat, weighing 4.6 kg, Astra blue staining, increased staining of Astra blue (arrows); (b) 17-year-old cat, weighing 2.75 kg, Astra blue staining, basic parallel-fibred structure retained, longitudinal chains of round-shaped cells (arrowhead)

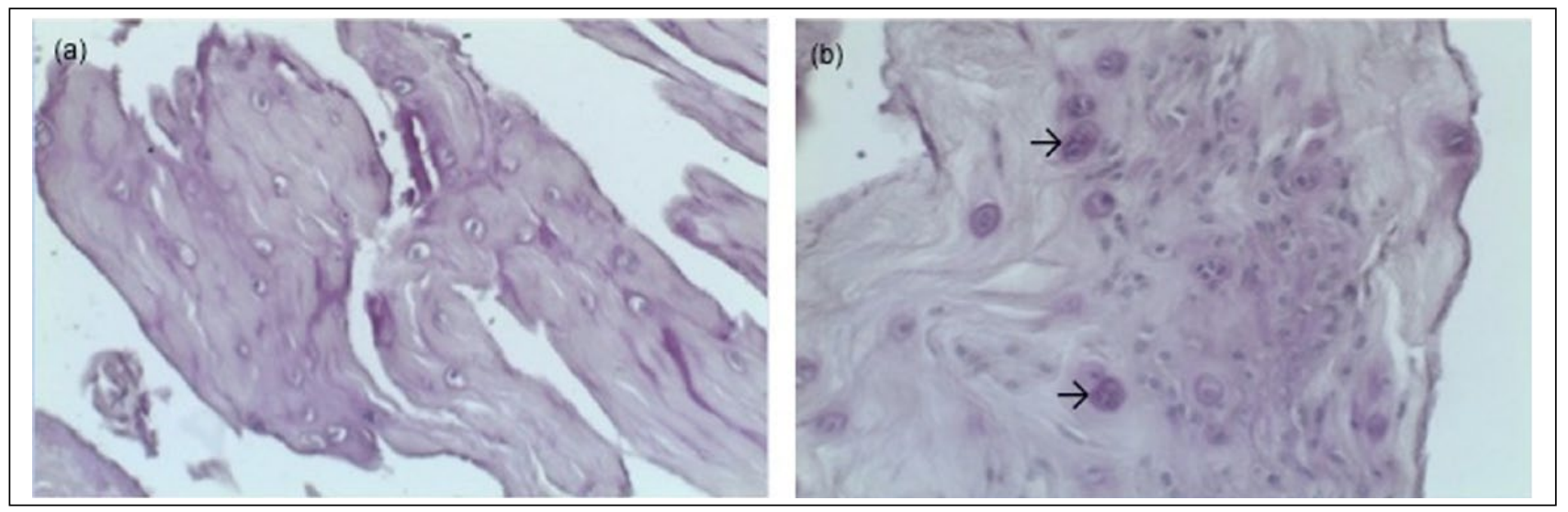

Figure 3 Microscopic anatomy of cranial cruciate ligaments (CCL) with a most likely traumatic rupture. (a) Ruptured CCL of a 7-year-old cat, weighing $3.3 \mathrm{~kg}$, periodic acid-Schiff (PAS) staining; (b) ruptured CCL of an 8-year-old cat, weighing $6.8 \mathrm{~kg}$, PAS staining, cartilage cells lying in small groups (arrows)

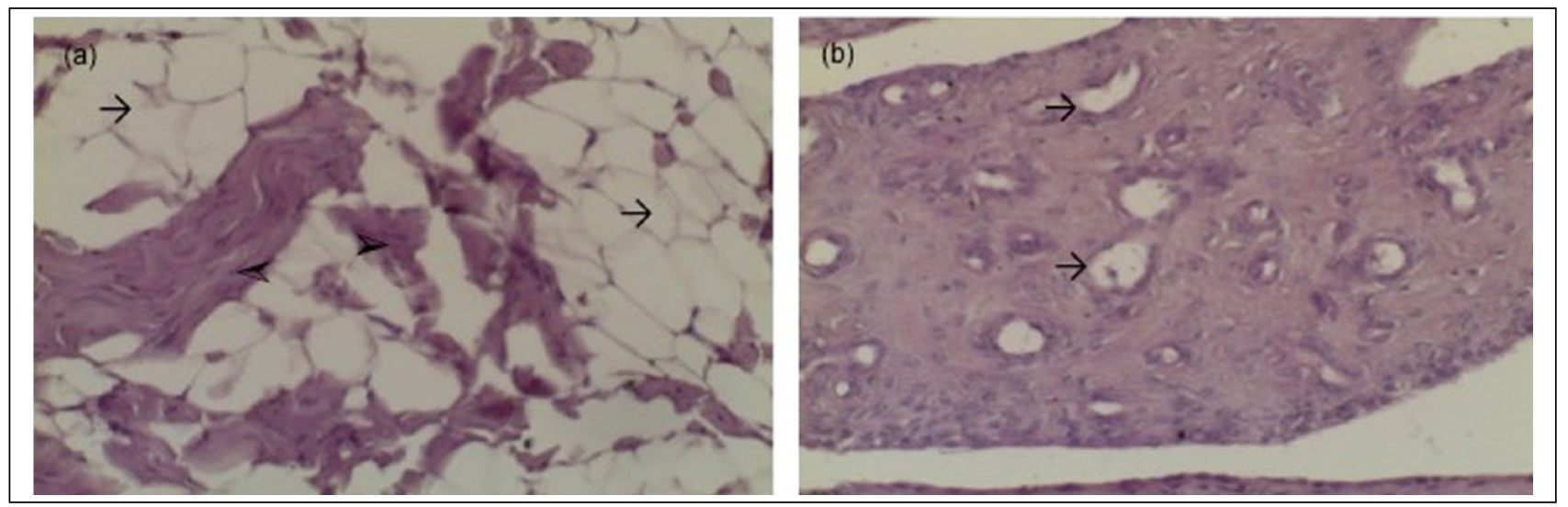

Figure 4 Microscopic anatomy of cranial cruciate ligaments (CCL) with a non-traumatic rupture. (a) Ruptured CCL of an 11 -year-old cat, weighing $4 \mathrm{~kg}$, replacement with adipose tissue (arrows), fragments of collagen fibres (arrowheads); (b) ruptured CCL of a 13-year-old cat, weighing $4.5 \mathrm{~kg}$, vascularisation (arrows) 


\section{Discussion}

Our hypothesis that degeneration of the CCL in cats similar to that in dogs ${ }^{12}$ - can lead to an isolated rupture during a minimal traumatic event was not confirmed by the results of our study.

Studies of CCLD have regularly demonstrated signs of degeneration (eg, lipid droplets and calcification) within the fibrocartilage cells, marked cartilage substance and cartilage cells with well-marked capsules. ${ }^{12}$ However, our study of cats showed less cartilage differentiation in CCLR and no evidence of either masking of collagen fibres or of lipid droplets or calcification. The macroscopic anatomy of intact CCLs from cats agreed with data reported by others in the current literature; 17,18 the collected findings indicate characteristics of physiological differentiation of fibrocartilage in tendons or ligaments. Moreover, similar results were reported from a study by Heintel of canine tendons. ${ }^{19}$

Fibrocartilaginous segments have a significantly lower tensile strength. ${ }^{19,20}$ Normally, this feature is compensated for by a greater cross-sectional area, without which the section is prone to rupture. ${ }^{20}$ Owing to the inherent anatomy of the stifle, development of a greater cross-sectional area is not possible. As the stifle is flexed, the cruciate ligaments begin to twist upon each other; this twisting action limits the amount of normal internal rotation of the tibia. ${ }^{21}$ The region where the CCL twists against the caudal cruciate ligament appears to be a focal area of compression in the CCL, caused by the forces of the opposing caudal cruciate ligament. ${ }^{10,22}$

Incorporation of fibrocartilage into the CCL leads to biomechanical weakening of the ligament. Formation of fibrocartilage in the middle of the CCL is often misclassified as a degenerative change. ${ }^{8-10,13}$ Certainly, degenerative changes within the fibrocartilage itself are possible; this has been evidenced by histological studies of canine $\mathrm{CCLs}^{9,12,23}$ An electron microscopy investigation of the canine cruciate ligament provided the first evidence of fibrocartilage in 1-month-old dogs. ${ }^{12}$ Our study used classic light microscopy techniques to study healthy cats and found neither evidence of fibrocartilage in young cats ( $<0.6$ years) nor loss of the typical ligament structure in cats of all ages $(<0.6-17$ years). These findings were distinct from those reported in the dog, which have generally shown a loss of the typical ligament structure starting between 2 and 5 years of age, accompanied by areas with shattered or ruptured collagen fibres. ${ }^{12}$

The differentiation degree of fibrocartilage is dependent on body weight. ${ }^{10,23}$ In our current study, the intact CCL of the second-heaviest cat $(7 \mathrm{~kg})$ showed early signs of fibrocartilage. This finding supports the thesis that more massive degenerative changes are associated with increasing body weight. ${ }^{9}$

Obesity is considered to be a risk factor for rupture of the CCL in cats. ${ }^{13,24,25}$ In contrast, in the current study, the body weight of 200 healthy cats (a random sampling) was statistically compared with cats with CCLR, and no statistical difference was found. Therefore, the assumption that cats with CCLR are heavier than healthy cats could not be confirmed.

Because rupture of the CCL is common in small dog breeds, ${ }^{26}$ the small body size and lower body weight in cats is not an adequate explanation for the apparently infrequent occurrence of this injury in the cat. ${ }^{24}$

In the current literature, the occurrence of fibrocartilage is equated with the presence of degeneration. ${ }^{13,25} \mathrm{In}$ a clinical setting, the term 'degeneration' denotes morphological dysfunction of an organ or organ system. ${ }^{27}$ Swelling and thickening of collagen fibres are considered as characteristic of degenerative changes of connective tissue. ${ }^{27}$ Although it does seem likely that fibrocartilage can develop pathologically within tendons and ligaments, our results indicated that fibrocartilage should be primarily viewed as a tissue that develops normally over the lifespan in response to compression.

One main limitation of our study is the inherent and uncontrollable inaccuracies of human recall (ie, the owners' recollections as to the cause of the trauma). Moreover, it is well recognised that owner observations of cats are generally less complete than those of dogs. Thus, the occurrence of a traumatic event cannot be excluded for either the indoor or outdoor cats in our study. In general, a traumatic event is assumed to be the main cause of CCLR in cats; ${ }^{1,25}$ however, the actual cause is not entirely clear in every case. ${ }^{13,16}$ In the current study, we found similar results: $78.9 \%$ of the cats had - according to their owners - CCLR due to traumatic event. The remaining $21.1 \%$ of the cats all resided indoors exclusively, and a traumatic event was denied by the owners.

There are different outcomes concerning the aetiology of CCLR in cats. In Tacke and Schimke's investigation, ${ }^{25}$ $80 \%$ of cats had a traumatic rupture of the CCL. Scavelli and Schrader reported that only $16 \%$ of the cats in their study had a CCLR following a traumatic event, with the cause of rupture being unclear for the remaining $84 \% .{ }^{24}$ The study by Harasen classified $52.9 \%$ of the cats as suffering from a trauma-related CCLR, with all other cats in the study $(47.1 \%)$ being described as residing indoors exclusively and having no trauma-induced anamnesis. ${ }^{13}$

A further limitation of the current investigation that should be considered when interpreting the results is the relatively small population of cats with CCLR and, in particular, of cats with a non-traumatic rupture. However, a review of the current and past literature showed similarly low case numbers. ${ }^{13,15,16,24,25,28}$ Although we are affiliated with two small animal surgery referral centres (Small Animal Clinic Hollabrunn and The Small Animal Surgical Center, Austria) and one general small animal clinic (Small Animal Clinic Korneuburg, Austria), the number of cats with CCLR was quite small (19 cats in total). The study by Scavelli and Schrader was based on a total of 18 cats that presented with a CCLR at their 
animal medical centre over a 5 year period;24 yet, in approximately the same 5 year period, 260 dogs underwent surgery for rupture of the CCL. At the Veterinary University of Giessen, only 45 cats presented with a CCLR in a period of 10 years..$^{25}$ While the small numbers of cats with CCLR is common, the current study would have nonetheless benefited from a larger population for greater accuracy of the statistical analysis.

\section{Conclusions}

Although both cats and dogs have limited compensation by a greater cross-sectional area to counter potential damage by flexion forces in the stifle, ${ }^{10,22}$ cats show a lower rate of CCLR. An essential reason for this is the lower amount of differentiation of fibrocartilage compared with the dog, which might be due to the relatively lower body weight of cats in general. Degenerative changes within the fibrocartilage, as described in the literature for the $\operatorname{dog}_{1}{ }^{12}$ were not seen in our study. Therefore, our results suggest that fibrocartilage in the CCL should be viewed as a physiological reaction to normal physiological forces in the ligament and should not be immediately classified as a degenerative change. Biomechanical studies of feline CCLs are necessary to confirm and expand upon our findings.

Supplementary material A spreadsheet describing effect size calculations.

Conflict of interest The authors declared no potential conflicts of interest with respect to the research, authorship, and/ or publication of this article.

Funding The authors received no financial support for the research, authorship, and/or publication of this article.

\section{References}

1 McLaughlin R. Surgical diseases of the feline stifle joint. Vet Clin North Am Small Anim Pract 2002; 32: 963-982.

2 Comerford EJ, Smith K and Hayashi K. Update on the aetiopathogenesis of canine cranial cruciate ligament disease. Vet Comp Orthop Traumatol 2011; 24: 91-98.

3 Brunnberg L. Klinische untersuchungen zur ätiologie und pathogenese der ruptur des ligamentum cruciatum craniale beim hund. Kleintierpraxis 1989; 34: 111-114.

4 Witsberger TH, Villamil JA, Schultz LG, et al. Prevalence of and risk factors for hip dysplasia and cranial cruciate ligament deficiency in dogs. J Am Vet Med Assoc 2008; 232: 1818-1824.

5 Whitehair JG, Vasseur PB and Willits NH. Epidemiology of cranial cruciate ligament rupture in dogs. J Am Vet Med Assoc 1993; 203: 1016-1019.

6 Benjamin M and Ralphs JR. Tendons and ligaments - an overview. Histol Histopathol 1997; 12: 1135-1144.

7 Chowdhury P, Matyas JR and Frank CB. The 'epiligament' of the rabbit medial collateral ligament: a quantitative morphological study. Connect Tissue Res 1991; 27: 33-50.
8 Zahm $\mathrm{H}$. Die Ligamenta decussata im gesunden und arthrotischen Kniegelenk des Hundes. Kleintierpraxis 1965; 10: 38-47.

9 Vasseur PB, Pool RR, Arnoczky SP, et al. Correlative biomechanical and histologic study of the cranial cruciate ligament in dogs. Am J Vet Res 1985; 46: 1842-1854.

10 Hayashi K, Frank JD, Dubinsky C, et al. Histologic changes in ruptured canine cranial cruciate ligament. Vet Surg 2003; 32: 269-277.

11 Ichinohe T, Kanno N, Harada Y, et al. Degenerative changes of the cranial cruciate ligament harvested from dogs with cranial cruciate ligament rupture. J Vet Med Sci 2015; 77: 761-770.

12 Reese $S$. Untersuchungen am intakten und rupturierten Ligamentum cruciatum craniale des Hundes. MS thesis, College of Veterinary Medicine, University of Berlin, 1995.

13 Harasen GLG. Feline cranial cruciate ligament rupture. Vet Comp Orthop Traumatol 2005; 18: 254-257.

14 Ruthrauff CM, Glerum LE and Gottfried SD. Incidence of meniscal injury in cats with cranial cruciate ligament ruptures. Can Vet J 2011; 52: 1106-1110.

15 Perry K and Fitzpatrick N. Tibial tuberosity advancement in two cats with cranial cruciate ligament deficiency. Vet Comp Orthop Traumatol 2010; 23: 196-202.

16 Matis U, Holz I and Brühschwein A. TPLO in the cat. Proceedings of the 3rd World Veterinary Orthopaedic Congress; 2010 Sept 15-18; Bologna, Italy, pp 145-146.

17 Prosé LP. Anatomy of the knee joint of the cat. Acta Anat 1984; 119: 40-48.

18 Vollmerhaus B, Waibl $\mathrm{H}$ and Roos H. Kniegelenk. In: Frewein J and Vollmerhaus B (eds). Anatomie von hund und katze. 1st ed. Hamburg, Berlin: Parey, 1994, pp 69-71.

19 Heintel KE. Untersuchungen zu biomechanischen Eigenschaften von gleit- und zugsehnen. MS thesis, College of Veterinary Medicine, LMU Munich, 2013.

20 Jopp I and Reese S. Morphological and biomechanical studies on the common calcaneal tendon in dogs. Vet Comp Orthop Traumatol 2009; 22: 119-124.

21 Arnoczky SP. Pathomechanics of cruciate ligament and meniscal injuries. In: Bojrab MJ (ed). Disease mechanisms in small animal surgery. 2nd ed. Philadelphia, PA: Lippincott Williams \& Wilkins, 1993, pp 766-771.

22 Hayashi K, Bhandal J, Rodriguez CO, et al. Vascular distribution in ruptured canine cranial cruciate ligament. Vet Surg 2011; 40: 198-203.

23 Albers J. Biomechanische Untersuchungen an der Bizepssehne des Hundes. MS thesis, College of Veterinary Medicine, LMU Munich, 2012.

24 Scavelli TD and Schrader SC. Nonsurgical management of rupture of the cranial cruciate ligament in $\mathbf{1 8}$ cats. J Am Vet Med Assoc 1987; 23: 337-340.

25 Tacke $S$ and Schimke E. Zur ruptur der ligamenta cruciata bei der katze. Kleintierpraxis 1995; 40: 341-350.

26 Harasen G. Canine cranial cruciate ligament rupture in profile: 2002-2007. Can Vet J 2008; 49: 193-194.

27 Dahmen G. Alterungs- und degenerationsveränderungen des bindegewebes in ihrer bedeutung für die klinik. Zeitschrift für Rheumaforschung 1964; 11/12: 393-405.

28 Schnabl E, Reese S, Lorinson K, et al. Measurement of tibial plateau angle in cats with and without cranial cruciate ligament rupture. Vet Comp Orthop Traumatol 2009; 22: 83-86. 